\title{
First-order reversal curve analysis of graded anisotropy FePtCu films
}

\author{
Valentina Bonanni, ${ }^{1, a)}$ Yeyu Fang, ${ }^{1}$ Randy K. Dumas, ${ }^{2}$ Chaolin Zha, ${ }^{1}$ Stefano Bonetti, ${ }^{1}$ \\ Josep Nogués, ${ }^{1,3}$ and Johan Åkerman ${ }^{1,2,6)}$ \\ ${ }^{1}$ Material Physics, Royal Institute of Technology (KTH), 16440 Kista, Sweden \\ ${ }^{2}$ Department of Physics, University of Gothenburg, 41296 Gothenburg, Sweden \\ ${ }^{3}$ Institució Catalana de Recerca $i$ Estudis Avançats (ICREA) and Centre d'Investigació en Nanociència $i$ \\ Nanotecnologia (ICN-CSIC), Campus Universitat Autònoma de Barcelona, 08193 Bellaterra, Spain
}

(Received 19 August 2010; accepted 24 October 2010; published online 15 November 2010)

\begin{abstract}
The reversal mechanisms of graded anisotropy $\mathrm{FePtCu}$ films have been investigated by alternating gradient magnetometer (AGM) and magneto-optical Kerr effect (MOKE) measurements with first-order reversal curve (FORC) techniques. The AGM-FORC analysis, which clearly shows the presence of soft and hard components, is unable to resolve how these phases are distributed throughout the film thickness. MOKE-FORC measurements, which preferentially probe the surface of the film, reveal that the soft components are indeed located toward the top surface. Combining AGM-FORC with the inherent surface sensitivity of MOKE-FORC analysis allows for a comprehensive analysis of heterogeneous systems such as graded materials. () 2010 American Institute of Physics. [doi:10.1063/1.3515907]
\end{abstract}

Graded anisotropy materials ${ }^{1,2}$ have joined the ranks of tilted $^{3,4}$ and exchange coupled composite ${ }^{5}$ (ECC) recording media as possible solutions to the magnetic recording trilemma, ${ }^{6,7}$ where simultaneously balancing the thermal stability and signal-to-noise ratio with the writability of a given bit is necessary. Energy-assisted recording techniques, such as heat-assisted magnetic recording ${ }^{8}$ and microwave-assisted magnetic recording, ${ }^{9}$ rely on adding energy to the media in order to temporarily surmount the switching barrier with modest applied fields. In graded materials, which are predicted to provide additional gains in writability over conventional bilayer hard/soft ECC media, the anisotropy is systematically varied such that the switching field is reduced by the low anisotropy layers, while the high anisotropy layers preserve thermal stability. There have been few reports, mostly based on rather thick multilayered structures, on the successful creation of graded materials. ${ }^{10-14}$ However, the realization of a continuous anisotropy gradient was recently achieved in properly annealed compositionally graded thin FePtCu films. ${ }^{15}$ In conjunction with the fabrication, the subsequent analysis of graded materials is not trivial and often requires complicated measurement apparatuses. For example, the inherent depth sensitivity of polarized neutron reflectivity measurements has recently been used to directly probe the anisotropy gradient of multilayered $\mathrm{Co} / \mathrm{Pd}$ samples. ${ }^{12,13}$

In this letter, we report on a relatively simple measurement and analysis technique that combines first-order reversal curve (FORC) ${ }^{16-22}$ measurements with the inherent surface sensitivity of the magneto-optical Kerr effect (MOKE) to analyze $\mathrm{FePtCu}$ films with a continuously graded anisotropy. In addition to providing a useful qualitative fingerprint of the magnetization reversal mechanisms, the FORC techniques are able to extract a wealth of quantitative information not readily accessible from standard major loop or remanence curve analysis. For example, information on

\footnotetext{
${ }^{a}$ Present address: Department of Applied Physics, Chalmers University of Technology, 41296 Gothenburg, Sweden.

${ }^{b)}$ Electronic mail: johan.akerman@physics.gu.se.
}

distributions of key magnetic parameters, interactions, and phase identification in a variety of bulk, thin film, and patterned systems has been shown. ${ }^{16-22}$ To date, however, FORC analysis has focused on measurements that encompass the entire magnetic volume. Graded materials, which are explicitly designed to exhibit magnetic properties that vary throughout their thickness, provide a unique opportunity to extend the capabilities of FORC with the surface sensitivity of MOKE.

The sample for this study consists of a $50 \mathrm{~nm}$ thick $\left(\mathrm{Fe}_{53} \mathrm{Pt}_{47}\right)_{100-x} \mathrm{Cu}_{x}(x=0-30)$ film where the $\mathrm{Cu}$ concentration is continuously varied from $\mathrm{Cu}$-rich $\left(\mathrm{Fe}_{53} \mathrm{Pt}_{47}\right)_{70} \mathrm{Cu}_{30}$ at the bottom to $\mathrm{Cu}$-free $\mathrm{Fe}_{53} \mathrm{Pt}_{47}$ at the top. In order to improve surface roughness, (111)-texture, and lower the chemical ordering temperature of the $\mathrm{L}_{0}$ phase, the $\mathrm{FePtCu}$ films are deposited on a bilayer of $\mathrm{Ta}(6 \mathrm{~nm}) / \mathrm{Pt}(3 \mathrm{~nm})$ and capped with $5 \mathrm{~nm}$ of Ta to prevent oxidation. ${ }^{23-25}$ The anisotropy gradient is then realized after thermal annealing at a suitable temperature and by exploiting the strong dependence of the A1 (low anisotropy) to $\mathrm{L}_{0}$ (high anisotropy) ordering temperature on the $\mathrm{Cu}$ content. ${ }^{26}$ The $\mathrm{Cu}$-rich regions transform from the as-deposited low anisotropy cubic $\mathrm{A} 1$ phase into the high anisotropy tetragonal $\mathrm{L}_{0}$ phase at a lower annealing temperature than the $\mathrm{Cu}$-poor regions, thus establishing a continuous anisotropy gradient through the thickness of the film. Separate pieces of the as-deposited film were then annealed at 300 and $400{ }^{\circ} \mathrm{C}$ for $35 \mathrm{~min}$ in vacuum. More detailed information about sample fabrication and structural analysis can be found in Ref. 15 .

Magnetic properties have been measured using an alternating gradient magnetometer (AGM) and a homebuilt MOKE setup in the longitudinal configuration using a diode laser $(\lambda=633 \mathrm{~nm})$ polarized perpendicular to the plane of incidence (s-polarization). The AGM measures the magnetic response of the entire film and gives an overall view of the magnetization reversal, whereas the MOKE measurements preferentially sample the surface of the film. We have estimated the penetration depth of light $(\sim 30 \mathrm{~nm})$, employing the optical properties of bulk FePt. ${ }^{27}$ All the measurements 


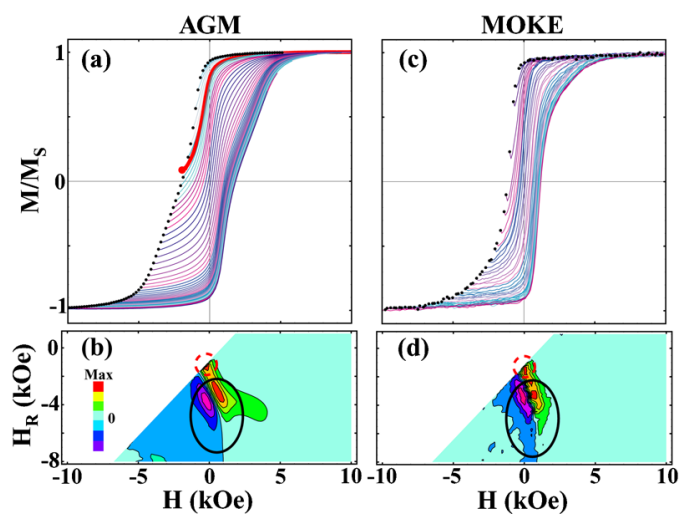

FIG. 1. (Color online) Families of FORCs for the graded FePtCu film annealed at $300{ }^{\circ} \mathrm{C}$ measured using the (a) AGM and (c) MOKE, where the first point of each FORC is shown by a dot. The corresponding FORC distributions are shown in (b) and (d), respectively. The bold FORC in (a) indicates the initial soft phase portion of the reversal and the dashed circle and solid oval highlight the soft and hard portions of the reversal, respectively.

are performed at room temperature with the field applied parallel to the film plane and along the same in-plane direction, given any potential in-plane anisotropy.

In addition to standard major loop and remanence curve analysis, both the AGM and MOKE are used to measure families of FORC curves using the procedure outlined in Refs. 16-22. The FORC distribution is then defined as a mixed second order derivative of the normalized magnetization over the applied field $H$ and the reversal field $H_{R}:{ }^{19}$ $\rho\left(H, H_{R}\right) \equiv-1 / 2\left[\left(\partial^{2} M\left(H, H_{R}\right) / M_{s}\right) /\left(\partial H \partial H_{R}\right)\right]$, which is then plotted against $\left(H, H_{R}\right)$ coordinates on a contour map. The FORC distribution provides a visual "fingerprint" by eliminating the purely reversible contributions to the reversal. For a given reversal field $H_{R}$, the magnetization is measured for increasing applied fields $H$ and therefore, $H \geq H_{R}$. Often it is better to have a one-dimensional visualization of the irreversibility by projecting the FORC distribution onto the $H_{R}$-axis. This is equivalent to an integration over the applied field $H: \int\left(\partial^{2} M\left(H, H_{R}\right) / \partial H \partial H_{R}\right) d H=\partial M\left(H_{R}\right) / \partial H_{R}$ and is termed a FORC-switching field distribution (FORCSFD), which can then be easily compared with the standard technique of taking the derivative of the dc-demagnetization curve to determine the dc-demagnetization switching field distribution (DCD-SFD): $\partial M_{r}\left(H_{R}\right) / \partial H_{R}$, where $M_{r}\left(H_{R}\right)$ is the zero-field magnetization, or remanence, after the application of a given reversal field $H_{R}$.

The family of FORCs and corresponding FORC distributions measured by AGM and MOKE are shown in Figs. 1(a) and 1(c) and Figs. 1(b) and 1(d), respectively. For the graded $\mathrm{FePtCu}$ film annealed at $300{ }^{\circ} \mathrm{C}$, the AGM major loop, seen as the outer boundary of the FORCs, shows a rather gradual reversal process and a coercivity of $1.98 \mathrm{kOe}$ [Fig. 1(a)]. A close inspection reveals that the major loop appears slightly pinched, which suggests the presence of both soft and hard phases. The AGM-DCD-SFD shows a large peak located at $H_{R}=-3.5 \mathrm{kOe}$ [Fig. 2(a) (solid squares)], which indicates that the majority of the switching processes occur for this reversal field. However, upon closer inspection a second and much smaller peak located at $H_{R}=-1.7 \mathrm{kOe}$ (indicated with an arrow) indicates the presence of soft phase switching. Interestingly, the soft phase only becomes fully evident in the full FORC analysis. The

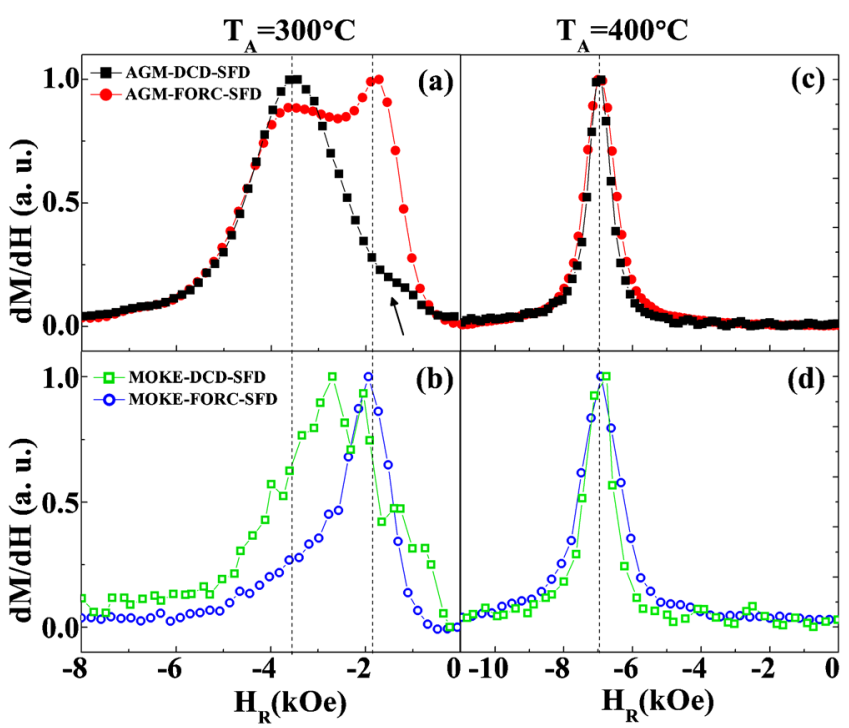

FIG. 2. (Color online) SFDs of the FePtCu film annealed at (a, b) 300 and (c, d) $400{ }^{\circ} \mathrm{C}$ calculated from (a, c) AGM and (b, d) MOKE measurements. The DCD-SFDs (solid and open squares) are calculated by taking the derivative of the DCD curve. The FORC-SFDs (solid and open circles) are the projection of the corresponding FORC distributions onto the $H_{R}$-axis. The arrow in (a) indicates the small peak discussed in the text. All distributions are normalized by their maximum values.

AGM-FORC distribution [Fig. 1(b)] shows two primary features. The first feature, highlighted with a dashed circle, is a sharp and localized peak located near the $H=H_{R}$ boundary and can be associated with a soft phase as it occurs for small $H_{R}$ values. The second feature, highlighted with a solid oval, is a negative-positive pair of features that extend downward parallel to the $H_{R}$-axis and can be associated with the hard phase. From a fingerprinting standpoint, this FORC distribution is reminiscent of those previously observed in soft/hard bilayer exchange springs. ${ }^{17}$ In order to better compare with the AGM-DCD-SFD, the AGM-FORC-SFD is calculated [Fig. 2(a) (solid circles)] and shows two distinct peaks. Unlike the AGM-DCD-SFD, the soft phase peak, located at $H_{R}=-1.7 \mathrm{kOe}$, is not only enhanced but also slightly larger than the hard phase peak located at $H_{R}=-3.5$ kOe. The significant overlap between the soft and hard peaks indicates that these phases are indeed highly coupled. An inspection of the minor loop bounded by the descending branch of the major loop and the highlighted FORC in Fig. 1(a) $\left(H_{R}=-1.7 \mathrm{kOe}\right)$ reveals a clear shift toward negative fields due to a significant exchange coupling to the hard phase. In fact, the striking differences between the AGM-DCD-SFD and AGM-FORC-SFD is in stark contrast to what has been previously observed in standard bilayer exchange spring systems where only minor differences were evident. ${ }^{17}$ The difference between the AGM-DCD-SFD and the AGM-FORC-SFD arises because the AGM-DCDSFD is only sensitive to switching events as they are manifested at the remanent state, whereas the AGM-FORC-SFD accounts for irreversibility along the entire reversal curve. During the soft phase reversal, the remanent state changes very little because the soft phase minor loop is significantly exchange shifted toward the left, which renders it virtually invisible in the AGM-DCD-SFD. However, the FORC analysis, which is sensitive to the total irreversibility present, is able to clearly resolve the soft and hard layers alike. It must be noted that while the AGM-FORC analysis is able to re- 
veal the soft/hard components, it is not able to directly probe how they are distributed through the film thickness.

In order to selectively probe the surface of the film, MOKE measurements are performed. The family of FORC curves measured using the MOKE is shown in Fig. 1(c). Clearly, the major loop exhibits a different reversal behavior as that observed using the AGM. The reversal begins with a sharp decrease in magnetization followed by a more gradual approach toward saturation. Most notably, the MOKE major loop shows a reduced coercivity of $1.28 \mathrm{kOe}$ as compared to the AGM measurements, which indicates that the softer portions of the sample are indeed near the surface, as designed. The MOKE-DCD-SFD is shown in Fig. 2(b) (open squares). While both the AGM and MOKE-DCD-SFDs reveal two peaks, two distinct differences are observed. First, the high anisotropy peak is shifted toward less negative reversal fields $\left(H_{R}=-2.7 \mathrm{kOe}\right)$. Second, the high and low anisotropy peaks now have comparable amplitudes. Both of these differences are consistent with the fact that the MOKE measurements preferentially probe the lower anisotropy layers near the surface. A comparison of the AGM and MOKE-FORC distributions, Figs. 1(b) and 1(d), respectively, reveals qualitatively similar features that correspond to the soft and hard phases. However, the key difference between the AGM and MOKEFORC distributions lies in the relative intensities of the peaks and valleys. This difference, which is not immediately observable from the contour plots, becomes clear in the projected data and the MOKE-FORC-SFD [Fig. 2(b) (open circles)]. The relative intensities of the negative and positive portions of the high anisotropy tail [Fig. 1(d)] are nearly identical in magnitude, leading to the virtual cancellation in the projected data. Clearly, the MOKE-FORC-SFD predominately shows the low anisotropy peak located at $H_{R}=$ $-1.7 \mathrm{kOe}$. As was also observed in the AGM-FORC-SFD [Fig. 2(a)], the MOKE-FORC-SFD is able to clearly resolve the soft phase as the total irreversibility during soft phase reversal is large. The MOKE-FORC-SFD is highly asymmetric and extends toward large negative $H_{R}$ values because, while the light preferentially samples the surface, some of the MOKE signal does emanate from deep within the film where the anisotropy is large.

For comparison, the AGM-DCD-SFD, AGM-FORCSFD, MOKE-DCD-SFD, and MOKE-FORC-SFD are measured for a sample annealed at $400{ }^{\circ} \mathrm{C}$, as shown in Figs. 2(c) and 2(d). We see that not only have the peaks moved toward more negative $H_{R}$ values, but also all four SFDs show significant overlap. The peak is located at a more negative $H_{R}$ value because the higher annealing temperature has increased the overall $\mathrm{L} 1_{0}$ ordering leading to an increase in anisotropy. As previously observed, ${ }^{15}$ the increased annealing temperature leads to significant interdiffusion, which results in a compositionally homogenous film with little to no anisotropy gradient and therefore nearly identical behavior in the AGM and MOKE measurements.

In summary, the reversal mechanisms of continuously graded $\mathrm{FePtCu}$ films have been investigated by both AGM and MOKE measurements with a FORC analysis technique. Unlike standard remanent curve analysis, the AGM-FORC analysis is able to clearly resolve the soft and hard components of the graded film as it probes the irreversibility along the entire reversal path. However, while the AGM-FORC analysis clearly shows the presence of both soft and hard phases, it is not able to resolve how they are distributed throughout the film thickness. MOKE-FORC measurements, which preferentially probe the surface of the film, reveal that the soft components are indeed toward the top of the film, as designed. In the fully annealed sample, the AGM and MOKE-FORC analyses reveal a highly homogeneous film. Combining the inherent surface sensitivity of MOKE with FORC techniques allows for a relatively easy, comprehensive, and powerful analysis tool for graded materials.

Support from The Swedish Foundation for strategic Research (SSF), The Swedish Research Council (VR), the Göran Gustafsson Foundation, and the Knut and Alice Wallenberg Foundation is gratefully acknowledged. V.B. thanks the Blanceflor Boncompagni-Ludovisi Foundation. J.N. thanks the Catalan DGR (Grant No. 2009SGR1292) and the Spanish MICINN (Grant No. MAT2010-20616-C02) projects for partial financial support. J. $\AA$. is a Royal Swedish Academy of Sciences Research Fellow supported by a grant from the Knut and Alice Wallenberg Foundation.

${ }^{1}$ D. Suess, Appl. Phys. Lett. 89, 113105 (2006).

${ }^{2}$ D. Suess, J. Fidler, G. Zimanyi, T. Schrefl, and P. Visscher, Appl. Phys. Lett. 92, 173111 (2008).

${ }^{3}$ K. Z. Gao and H. N. Bertram, IEEE Trans. Magn. 38, 3675 (2002).

${ }^{4}$ J. P. Wang, Nature Mater. 4, 191 (2005).

${ }^{5}$ R. H. Victora and X. Shen, IEEE Trans. Magn. 41, 537 (2005).

${ }^{6}$ M. H. Kryder and R. W. Gustafson, J. Magn. Magn. Mater. 287, 449 (2005).

${ }^{7}$ H. J. Richter, IEEE Trans. Magn. 35, 2790 (1999).

${ }^{8}$ T. W. McDaniel, J. Phys.: Condens. Matter 17, R315 (2005).

${ }^{9}$ J. G. Zhu, X. C. Zhu, and Y. H. Tang, IEEE Trans. Magn. 44, 125 (2008).

${ }^{10}$ V. Alexandrakis, D. Niarchos, K. Mergia, J. Lee, J. Fidler, and I. Panagiotopoulos, J. Appl. Phys. 107, 013903 (2010).

${ }^{11}$ D. Goll, A. Breitling, L. Gu, P. A. van Aken, and W. Sigle, J. Appl. Phys. 104, 083903 (2008).

${ }^{12}$ B. J. Kirby, J. E. Davies, K. Liu, S. M. Watson, G. T. Zimanyi, R. D. Shull, P. A. Kienzle, and J. A. Borchers, Phys. Rev. B 81, 100405 (2010).

${ }^{13}$ B. J. Kirby, S. M. Watson, J. E. Davies, G. T. Zimanyi, K. Liu, R. D. Shull, and J. A. Borchers, J. Appl. Phys. 105, 07 C929 (2009).

${ }^{14}$ T. J. Zhou, B. C. Lim, and B. Liu, Appl. Phys. Lett. 94, 152505 (2009).

${ }^{15}$ C. L. Zha, R. K. Dumas, Y. Y. Fang, V. Bonanni, J. Nogués, and J. Åkerman, Appl. Phys. Lett. 97, 182504 (2010).

${ }^{16}$ J. E. Davies, O. Hellwig, E. E. Fullerton, G. Denbeaux, J. B. Kortright, and K. Liu, Phys. Rev. B 70, 224434 (2004).

${ }^{17}$ J. E. Davies, O. Hellwig, E. E. Fullerton, J. S. Jiang, S. D. Bader, G. T. Zimanyi, and K. Liu, Appl. Phys. Lett. 86, 262503 (2005).

${ }^{18}$ R. K. Dumas, C.-P. Li, I. V. Roshchin, I. K. Schuller, and K. Liu, Phys. Rev. B 75, 134405 (2007).

${ }^{19}$ C. R. Pike, A. P. Roberts, and K. L. Verosub, J. Appl. Phys. 85, 6660 (1999)

${ }^{20}$ J. E. Davies, J. Wu, C. Leighton, and K. Liu, Phys. Rev. B 72, 134419 (2005).

${ }^{21}$ R. K. Dumas, T. Gredig, C. P. Li, I. K. Schuller, and K. Liu, Phys. Rev. B 80, 014416 (2009).

${ }^{22}$ R. K. Dumas, K. Liu, C. P. Li, I. V. Roshchin, and I. K. Schuller, Appl. Phys. Lett. 91, 202501 (2007).

${ }^{23}$ C. L. Zha, J. Persson, S. Bonetti, Y. Y. Fang, and J. Åkerman, Appl. Phys Lett. 94, 163108 (2009).

${ }^{24}$ C. L. Zha, Y. Y. Fang, J. Nogues, and J. Åkerman, J. Appl. Phys. 106, 053909 (2009).

${ }^{25}$ C. L. Zha, S. Bonetti, J. Persson, Y. Zhou, and J. Åkerman, J. Appl. Phys. 105, 07E910 (2009).

${ }^{26}$ T. Maeda, T. Kai, A. Kikitsu, T. Nagase, and J.-i. Akiyama, Appl. Phys. Lett. 80, 2147 (2002).

${ }^{27}$ A. S. Sabau, P. B. Kadolkar, R. B. Dinwiddie, R. D. Ott, and C. A. Blue, Metall. Mater. Trans. A 38, 788 (2007). 